\title{
A GENERALISATION OF LORENTZ-EQUIVALENGE IN A SUBSTRATUM THEORY
}

\author{
S. J, PROKHOVNIK
}

(Received 7 April 1965)

\begin{abstract}
It has been shown that the assumption of a specified cosmological substratum leads to the Lorentz-equivalence of observers whose uniform motion in the substratum lies along a common straight line. The result is generalised for any pair of unaccelerated observers thus confirming that only the relative velocity of such observers is relevant in relating their observations of an event.
\end{abstract}

In a previous communication by the author [1], it was shown that the assumption of a cosmological substratum provides a sufficient basis for the operation of Einstein's principles and the associated Special Relativity Theory. Distinction was made between fundamental observers to whom the universe appears isotropic and moving observers to whom it does not so appear; and it was assumed, after McCrea [2], that light-propagation also is isotropic with respect to the fundamental observers. The resulting anisotropy with respect to moving observers led to a number of consequences.

Thus consider an observer $A$ (associated with a reference frame $I_{A}$ ) receding from a fundamental observer $S$ (associated with $I_{S}$ ) with relative velocity $u$, as measured according to Einstein's conventions by either observer. Then it was shown in [1] that an angle made with the direction of relative motion has different measures, $\theta$ and $\theta_{A}$, in $I_{S}$ and $I_{A}$ respectively, the relation between these measures being

$$
\tan \theta=\beta \tan \theta_{A}
$$

where

$$
\beta=\left(1-u^{2} / c^{2}\right)^{-\frac{1}{2}} .
$$

Imagine further that $A$ and $S$ measure time with similar clocks synchronised at the instant of their spatial coincidence; then it follows, as in [1], that $A$ 's Einstein measures, $\left(x_{A}, y_{A}, z_{A}, t_{A}\right)$ of the co-ordinates of an event, are related to the corresponding measures, $\left(x_{S}, y_{S}, z_{S}, t_{S}\right)$ according to $S$, by the Lorentz transformation

$$
\begin{aligned}
x_{A} & =\beta\left(x_{S}-u t_{S}\right), \quad y_{A}=y_{S}, \quad z_{A}=z_{S}, \\
t_{A} & =\beta\left(t_{S}-u x_{S} / c^{2}\right) .
\end{aligned}
$$

On account of its group character (in particular, its transitive prop- 
erty), the transformation (2) extends immediately to the corresponding observations of two moving observers with different velocities in the same straight line relative to the basic system $I_{S}$. It will now be shown that the Lorentz-equivalence of moving observers is not limited to the above case but applies in general to any pair of unaccelerated observers.

We will consider in the first place the case of two uniformly-moving observers, $A$ and $B$, whose paths in $I_{S}$ intersect at a point $O$ and who observe an event at $E$ coplanar with $A, B$ and $O$. In order to relate the $I_{A}$ and $I_{B}$ co-ordinates of the event we will consider also the corresponding Einstein measures of an observer, $S$, stationary at $O$, and of another observer $P$ who moves (with respect to $I_{S}$ ) in a direction normal to the direction of $A$ to $B$ such that he remains always collinear with these observers. We assume that the four observers employ similar clocks which were synchronised when they were together at $O$.

Since light propagation is isotropic only with respect to $I_{S}$ we will employ it as a basic frame of reference. Figure 1 depicts the paths and relative positions, with reference to $I_{S}$, of the observers and the event; the $I_{S}$ measure of the space interval $O E$ is denoted by $r$, of the angle $P O A$ by $\phi$, of the angle $A O E$ by $\theta$ and of the angle $B A O$ by $\psi, \psi$ and $\phi$ being complementary. The velocity of $A$ in $I_{S}$ is denoted by $u$, this being identical to the relative velocity of $A$ and $S$ as measured by either observer.

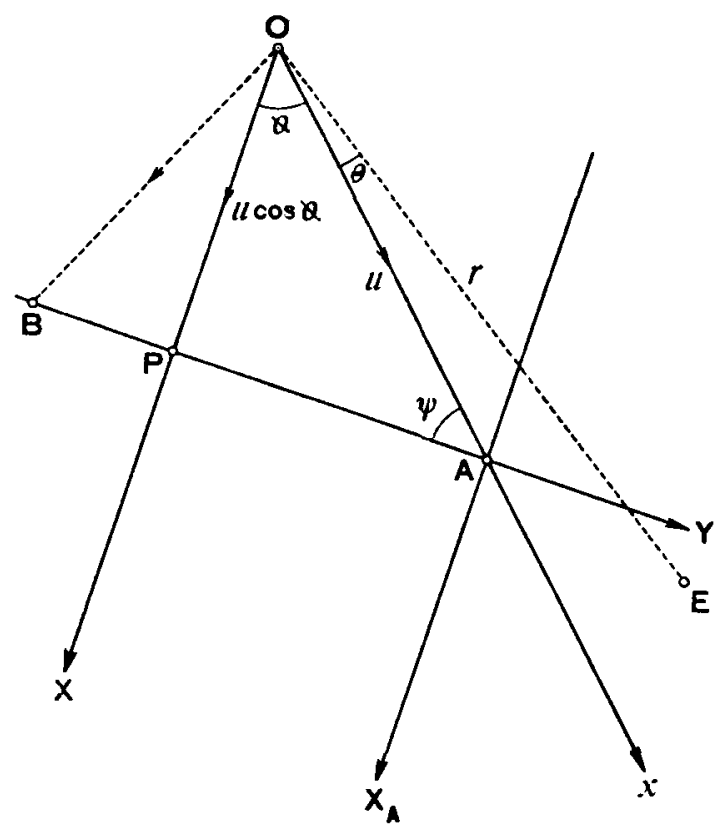

Figure 1. Representation of axes, velocities, angles and the distance $O E$, according to an observer at $O$ stationary in $I_{s}$. 
The various frames of reference may be based on a common $X$-axis for $S$ (at $O$ ) and $P$, and a common $x$-axis for $S$ and $A$, their origins being the locations of the various observers. Let the respective $Y$ - and $y$-axes lie in the common plane, as in the figure, the respective $Z$ - and $z$-axes being normal to the plane. We note that the $Y$-axis through $P$ is common to both $A$ and $B$.

The relation between the respective Einstein measures $\left(x_{A}, y_{A}, z_{A}, t_{A}\right)$ and $\left(x_{S}, y_{S}, z_{S}, t_{S}\right)$, according to $A$ and $S$, of the co-ordinates of the event is given by the transformation (2) where, in the present situation,

$$
x_{S}=r \cos \theta, \quad y_{S}=r \sin \theta, \quad z_{S}=O .
$$

The corresponding measures, $\left(X_{P}, Y_{P}, Z_{P}, t_{P}\right)$ and $\left(X_{S}, Y_{S}, Z_{S}, t_{S}\right)$, by $P$ and $S$, whose relative velocity along their common $X$-axis is $u \cos \phi$, are similarly related by

$$
\begin{aligned}
X_{P} & =\beta_{1}\left(X_{S}-u t_{S} \cos \phi\right), \quad Y_{P}=Y_{S}, \quad Z_{P}=Z_{S}, \\
t_{P} & =\beta_{1}\left[t_{S}-\left(u X_{S} \cos \phi\right) / c^{2}\right], \\
\text { where } \quad \beta_{1} & =\left[1-(u \cos \phi)^{2} / c^{2}\right]^{-\frac{1}{2}}=\left[1-(u \sin \psi)^{2} / c^{2}\right]^{-\frac{1}{2}} \\
\text { and } \quad X_{S} & =r \cos (\theta+\phi), \quad Y_{S}=r \sin (\theta+\phi), \quad Z_{S}=0 .
\end{aligned}
$$

We can now relate $A$ 's measures with $P$ 's by expressing the former in terms of a reference frame based on the $X$-and $Y$-directions. This requires a rotation of axes involving the angle $\psi_{A}-A$ 's measure of the angle $B A O$. $\psi_{A}$ is related to $\psi$ according to (1) which is equivalent to

$$
\begin{aligned}
& \beta_{1} \cos \psi=\cos \psi_{A} \\
& \beta_{1} \sin \psi=\beta \sin \psi_{A} .
\end{aligned}
$$

It follows that the Einstein measure, $v$, according to $A$ or $P$, of their mutual relative velocity along their common $Y$-axis is

$$
\begin{aligned}
v & =u \cos \psi_{A}=\beta_{1} u \cos \psi \\
& =\beta_{1} u \sin \phi
\end{aligned}
$$

Carrying out the rotation and using (5), $A$ 's measures $\left(X_{A}, Y_{A}, Z_{A}, t_{A}\right)$ and $\left(x_{A}, y_{A}, z_{A}, t_{A}\right)$ are related by

$$
\begin{aligned}
& X_{A}=x_{A} \sin \psi_{A}-y_{A} \cos \psi_{A}=\left(\beta_{1} / \beta\right) x_{A} \sin \psi-\beta_{1} y_{A} \cos \psi, \\
& Y_{A}=x_{A} \cos \psi_{A}+y_{A} \sin \psi_{A}=\beta_{1} x_{A} \cos \psi+\left(\beta_{1} / \beta\right) y_{A} \sin \psi, \\
& Z_{A}=z_{A}, \text { and } t_{A} \text { is the same for both reference frames. }
\end{aligned}
$$

We can now relate $A$ 's and $P$ 's measures in terms of their relative velocity, $v$. Thus combining (2), (3) and (4) 


$$
\begin{aligned}
t_{A} & =\beta\left[t_{S}-(u r \cos \theta) / c^{2}\right] \\
& =\beta\left[t_{P} / \beta_{1}+\left(u r / c^{2}\right) \cos (\theta+\phi) \cos \phi-\left(u r / c^{2}\right) \cos \theta\right] \\
& =\left(\beta / \beta_{1}\right)\left[t_{P}-\left(\beta_{1} u r / c^{2}\right) \sin \phi \sin (\theta+\phi)\right]
\end{aligned}
$$

Hence using (4) again and also (6), and noting that $\beta / \beta_{1}=\left(1-v^{2} / c^{2}\right)^{-1}=$ $\beta_{A P}$, we obtain

$$
t_{A}=\beta_{A P}\left(t_{P}-v Y_{P} / c^{2}\right)
$$

Similarly invoking (2), (3), (4) and (6) in (7), we obtain

$$
\begin{aligned}
Y_{A} & =\beta_{A P}\left(Y_{P}-v t_{P}\right), \\
X_{A} & =X_{P}, \\
Z_{A} & =Z_{P} .
\end{aligned}
$$

The results (8) and (9) demonstrate the Lorentz-equivalence of observers $A$ and $P$. Clearly a similar equivalence applies in respect to the observers $B$ and $P$; hence, since $A, P$ and $B$ are always co-linear (both in a relative sense and also in respect to the basic reference frame $I_{S}$ ), it follows on invoking the transitive property of the Lorentz transformation that $A$ and $B$ are therefore also Lorentz-equivalent. It is seen that only the relative motion of a pair of observers (such as $A$ and $P$ or $A$ and $B$ ) is relevant in relating their Einstein observations of an event.

In the case where the event $E$ is not coplanar with $A, B$ and $O$, its $z$ (or $Z$ )-coordinate will of course not be uniformly zero. However, the direction of the $z$ (or $Z$ )-axes is normal to the velocities in $I_{S}$ of each of these observers and hence, as shown in [1], the $z$ (or $Z$ )-coordinate of the event will still have the same Einstein measure for each of these observers, in terms of the reference-frames chosen above.

Finally we can generalise the result for unaccelerated observers whose paths do not intersect. For consider such a case involving our observer $A$, associated with the reference frame $I_{A}$, and a second observer $B^{\prime}$, associated with the reference frame $I_{B}$ so that $B^{\prime}$ and $B$ are at relative rest and hence equivalent in the Galileian sense - that is they can be considered Lorentzequivalent with zero relative velocity. The $I_{S}$ paths of $A$ and $B^{\prime}$ never intersect but those of $A$ and $B$ do; hence since $A$ and $B$ are Lorentz-equivalent in terms of a common zero-time, it follows that so are $A$ and $B^{\prime}$ provided that the clock employed by $B^{\prime}$ is synchronous with $B^{\prime}$ 's clock according to Einstein's synchronism convention.

Thus the assumption of the cosmological substratum defined in [1] leads to the system outlined by Einstein where only relative velocities are observable or relevant to the relating of observations of an event. The velocity of an observer relative to the substratum has relevance only to 
astronomical phenomena which are isotropic with respect to the basic reference frame, for instance the Doppler red-shift in the spectra of light from distant galaxies.

\section{References}

[1] Prokhovnik, S. J., "Neo-Lorentzian Relativity", J. Aust. Math. Soc. 5 (1965), 273-284.

[2] McCrea, W. H., 'Some Current Problems in Cosmology', Proc. Math. Soc. Univ. Southampion 5 (1962), 15.

School of Mathematics

University of New South Wales 